Case Report

\title{
Pleomorphic Adenoma of the Salivary Gland in Two Prairie Dogs (Cynomys ludovicianus)
}

\author{
Kiyokazu Ozaki ${ }^{1}$ and Isao Narama ${ }^{1}$ \\ ${ }^{1}$ Research Institute of Drug Safety, Setsunan University, 45-1 Nagaotohge-cho, Hirakata, Osaka 573-0101, Japan
}

\begin{abstract}
Two masses adjacent to the salivary glands of two Prairie dogs which were surgically removed and examined histopathologically as well as immunohistochemically, were diagnosed as pleomorphic adenomas. Histologically, each mass had a thin fibrous capsule, compressed surrounding tissue, and was lobulated by delicate connective tissue. The lobules mainly consisted of a solid growth of small ovoid- to spindle-shaped cells, and contained small duct or tubule lined with monolayered cuboidal epithelial cells. The amount of connective tissue stroma varied in different parts of the tumor mass, accompanied occasionally by a myxomatous stroma-rich area. Tumor cells in the solid growth area had indistinct cell borders, and usually showed spindle-like features resembling myoepithelial cells. Mitosis was prominent, whereas cellular pleomorphism was not seen. Tumor cells forming the tubular structure were positive for cytokeratin 19 and negative for smooth muscle actin (SMA), while the ovoid- to spindle-shaped tumor cells of the solid growth area were positive for SMA and negative for cytokeratin 19. Our results indicated that immunostaining was a useful and powerful method for the diagnosis of cell differentiation of salivary gland tumor cells. (J Toxicol Pathol 2003; 16: 171-173)
\end{abstract}

Key words: animal, immunohistochemistry, pleomorphic adenoma, salivary gland, tumor

Spontaneous salivary gland tumors were rare in the experimental rodent ${ }^{1}$. Therefore, histopathological classifications of the tumor in the rodent salivary gland were very simple compared with those of the human ${ }^{2,3}$. Pleomorphic adenoma is one of the most common neoplasms of human salivary gland, and characterized by the proliferation of morphological two types of tumor cells consisting of luminal and myoepithelial cells ${ }^{3}$. However, the term pleomorphic adenoma has not been used for the classification of rodent tumor ${ }^{2}$.

We encountered two cases of salivary gland tumor in the Prairie dog (Cynomys ludovicianus), a member of the family Rodentia. Prairie dogs had not been used for toxicological study, but we reported the morphological and immunohistochemical characters of the salivary gland tumors, thinking it might be useful for consideration of the diagnostic criteria for rare spontaneous salivary gland neoplasms in rats and mice ${ }^{1}$.

The tumors, which were surgically removed from a 2 year-old female (No. 1) Prairie dog and a female of unknown age (No. 2), were located in the cervical area. The masses

Received: 3 April 2003, Accepted: 10 July 2003

Mailing address: Kiyokazu Ozaki, Research Institute of Drug Safety, Setsunan University, 45-1 Nagaotohge-cho, Hirakata, Osaka

573-0101, Japan

TEL: 81-72-866-3162 Fax: 81-72-867-2370

E-mail: ozaki@pharm.setsunan.ac.jp were solitary and well demarcated without any adhesion to surrounding organs. They were both fixed in $10 \%$ neutral buffered formalin and embedded in paraffin, and sections were stained with hematoxylin and eosin, PAS reaction, and alcian blue with/without hyaluronidase digestion. For immunohistochemistry, antibodies to cytokeratin $14(\times 100$, monoclonal, Biomeda, USA), cytokeratin $19(\times 100$, monoclonal, Boehringer Mannheim Biochemica, Germany), human vimentin ( $\times 100$, monoclonal; DAKO, Denmark), and human $\alpha$ smooth muscle actin (SMA) $(\times 100$, monoclonal; Novocastra, UK) were used with simple stain rat MAX-PO (multi) (Nichirei, Japan).

The masses were $20 \times 15 \times 10 \mathrm{~mm}$ (No. 1) (Fig. 1) and $25 \times 15 \times 10 \mathrm{~mm}$ (No. 2) respectively, in size, and were whitecolored and firm. The cut surface was also white and firm, including some cystic areas varying in size.

Histologically, the two tumors showed a very similar structure. Each mass had a thin fibrous capsule, compressed surrounding tissue, and was lobulated by thin connective tissue (Fig. 2). The lobules mainly consisted of a solid growth of small ovoid to spindle cells, and contained small ducts or tubules lined by monolayered cuboidal epithelial cells (Fig. 3). A rosette formation was also seen in some epithelial cells. Varying amounts of connective tissue stroma had formed in the lobules and some areas were myxomatous or hemorrhagic. In some myxomatous areas, tubular and trabecular structures were prominent with a cystic dilated lumen (Fig. 4). These tubules were lined by a 


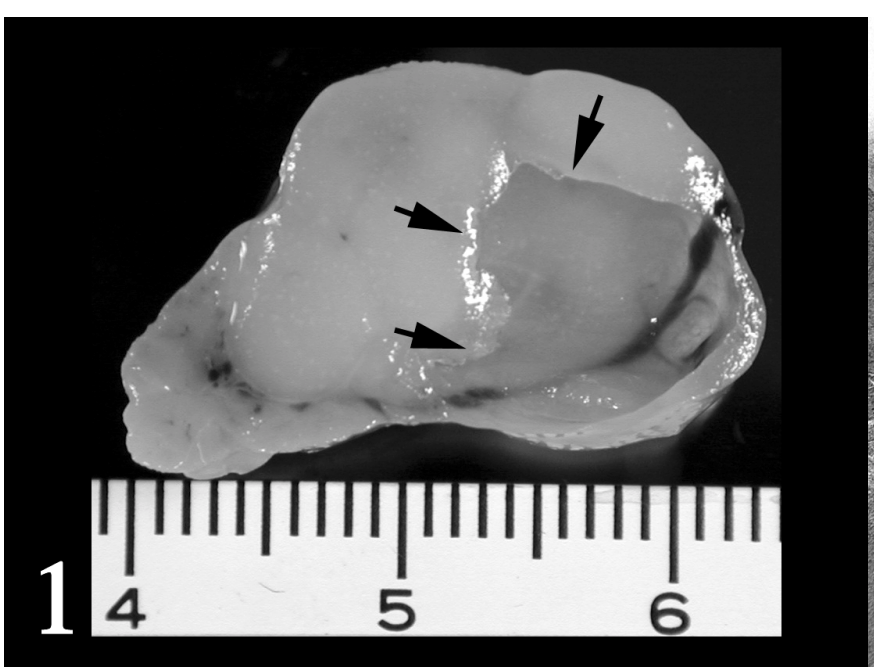

Fig. 1. Gross specimen of case \#1. The cut surface is white and firm, and includes a large cyst. The mass surrounding the salivary gland is compressed (arrows).

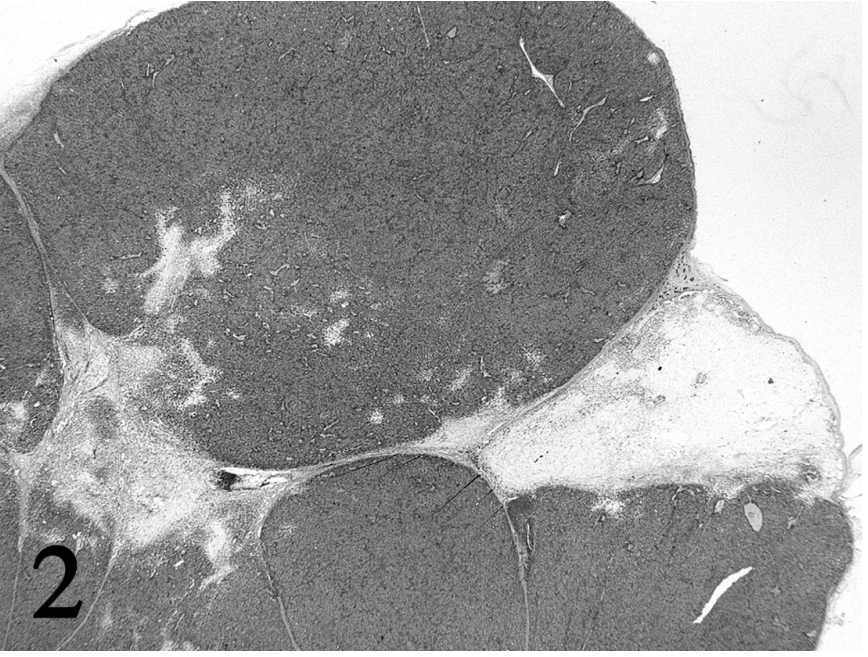

Fig. 2. Scanning magnification view of case \#2. The mass has a thin fibrous capsule, and is lobulated by connective tissue. Lobules mainly consist of a solid growth area, but occasionally contain myxomatous stroma.

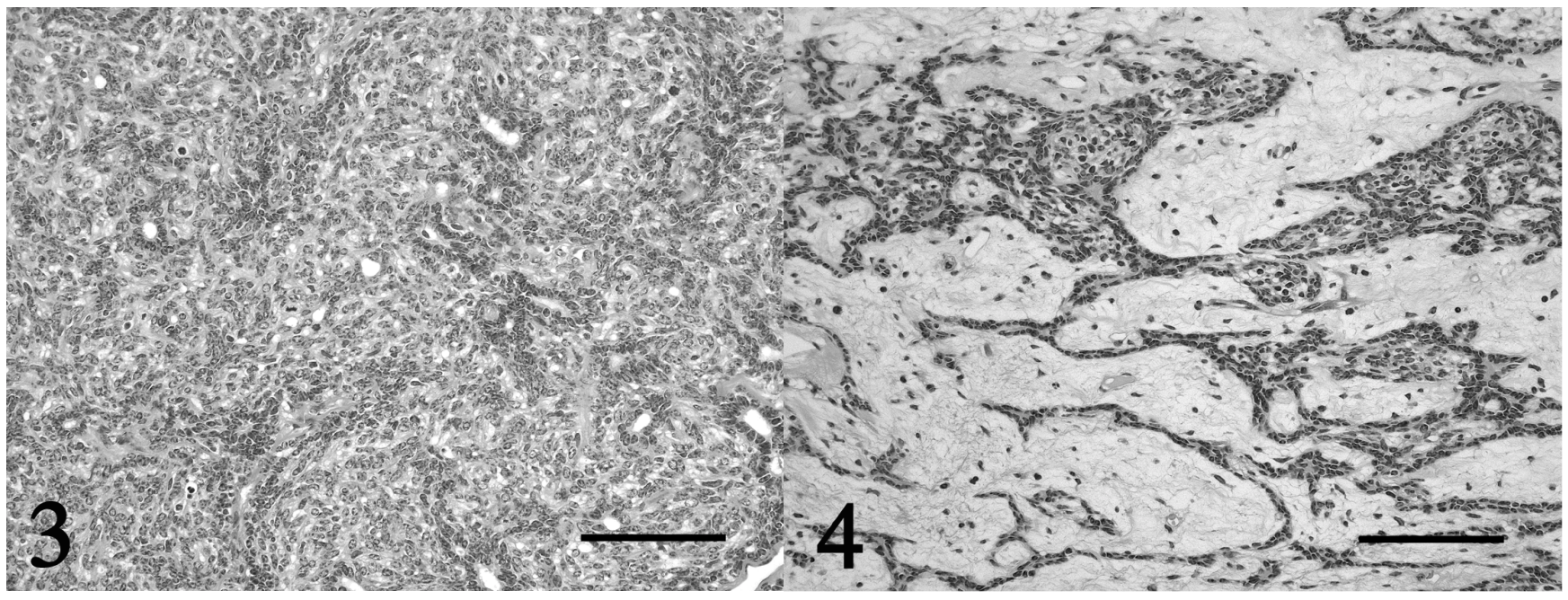

Fig. 3. Case $\# 2$. Lobules mainly consist of a solid growth of small ovoid- to spindle-shaped cells, including small ducts. HE. $\mathrm{Bar}=200 \mu \mathrm{m}$.

single cuboidal- to flattened- epithelium and often contained blood in the lumen. In some areas, the broader eosinophilic dense connective tissue stroma was apparent, and peripheral nuclear palisading was seen in some tumor lobules. Capsular invasion was not observed. Tumor cells in solid growth areas had an indistinct cell border and were usually spindle-shaped having a small elongated nucleus with clumped chromatin, resembling to those of myoepithelial cells. Mitoses were prominent in solid growth areas, whereas neither bizarre mitotic figures nor significant cellular pleomorphism was evident. Myxomatous stroma area was positive for alcian blue $(\mathrm{pH} \mathrm{2.5)}$ and weakly
Fig. 4. Case \#2. The trabecular structure is prominent in a myxomatous area. HE. Bar $=200 \mu \mathrm{m}$.

positive for PAS reaction. After hyaluronidase treatment, the positive reaction was disappeared. Some eosinophilic materials in the tubular lumen reacted positively with alcian blue ( $\mathrm{pH} 2.5)$.

In normal salivary glands, the ductal luminal epithelium was positive for cytokeratin 19 but rarely positive for cytokeratin 14. Myoepithelial cells just beneath the luminal epithelial cells were positive for SMA and positive for cytokeratin 14 in some areas. In the present cases, the tubular epithelium in solid areas was positive for cytokeratin 19 (Fig. 5) and negative for cytokeratin 14. In addition, tumor cells in solid areas were positive for SMA (Fig. 6) and 


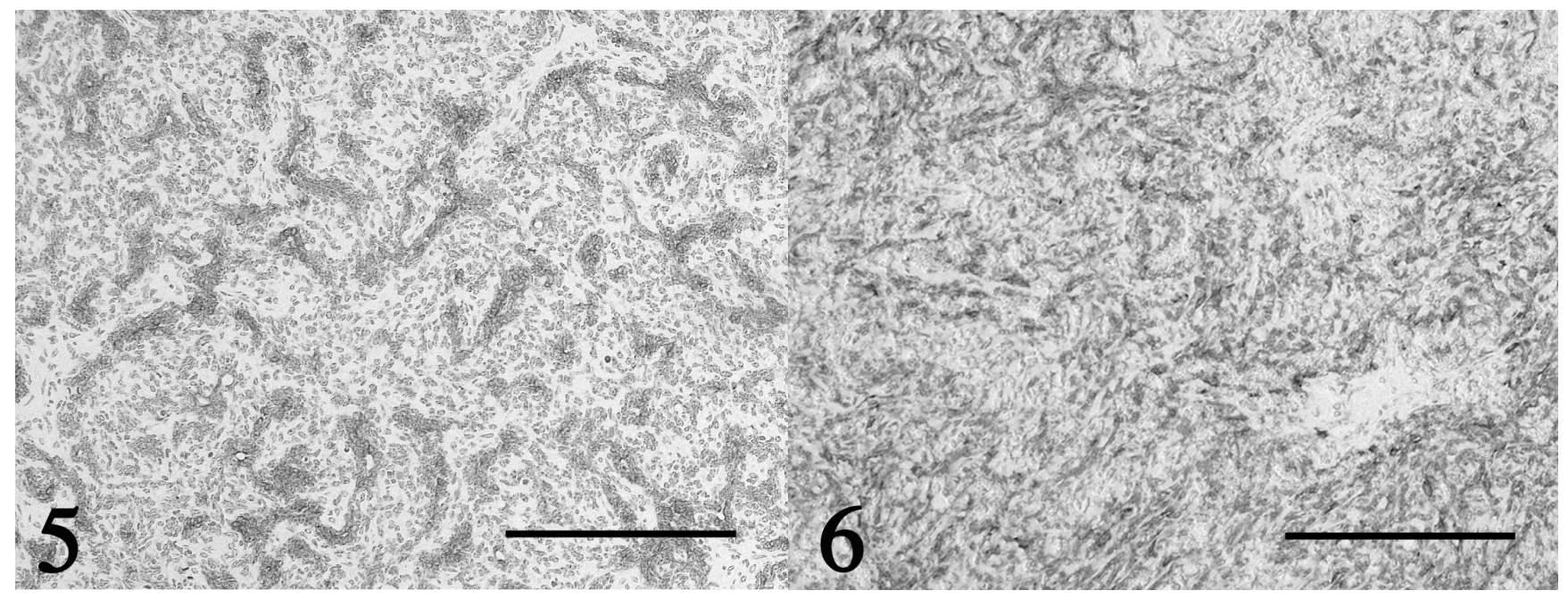

Fig. 5. Case \#2. Tubular epithelium in the solid area is positive for cytokeratin 19, but spindle cells are negative. Immunostaining for cytokeratin 19. Bar $=200 \mu \mathrm{m}$.

vimentin, and negative for cytokeratin 14. However, tubular epithelial cells in myxomatous areas were positive for both cytokeratin 19 and SMA.

The morphological and immunohistochemical characteristics of tubular and solid tumor cells in the present two cases were identical to those in the normal tubular luminal cells and myoepithelium of the salivary gland, respectively ${ }^{4}$. These characteristics strongly suggest that the tumor originated from both the luminal epithelium and myoepithelium. In addition, since the present cases had varying amounts of extracellular stroma, they were diagnosed as pleomorphic adenomas.

The differentiation between the benign and malignant nature of these tumors was very difficult because of two contrary phenomena, i.e., a high growth rate suggested by the high mitotic rate, and a lack of cell atypia. However, this tumor was well circumscribed and never showed any other evidence of malignancy such as coagulative necrosis, atypical mitotic figures, or cellular pleomorphism. According to the criteria for human pleomorphic adenoma ${ }^{3}$, these characters were consistent with a benign nature.

For the differential diagnosis of pleomorphic adenoma, it must be established that the present cases were not basal cell adenoma/carcinoma or adenocarcinoma. Basal cell adenoma/carcinoma is composed of cytokeratin14-positive basaloid cells, showing a solid, alveolar and ductular growth pattern $^{5}$, and often exhibiting characteristic nuclear palisading at the periphery. However, it does not include myxomatous stroma and is also positive for cytokeratin $14^{5}$. Since tumor cells of basal cell adenoma/carcinoma are mainly composed of basaloid cells, this tumor was unlikely to belong to that category. Adenocarcinomas of the mouse were reported to have a structure very similar to those of the present $\operatorname{cases}^{2,6}$. The solid growth area in our cases was
Fig. 6. Case \#2. Tubular epithelium in the solid area is negative for SMA, but spindle cells are positive. Immunostaining for SMA. $\mathrm{Bar}=200 \mu \mathrm{m}$

unlikely to be composed of poorly differentiated luminal cells, since tumor cells in the solid area were uniformly positive for SMA and vimentin, but negative for cytokeratin 14 and 19. The immunohistochemical characteristics of those spindle cells strongly suggested that they had originated from myoepithelial cells. Additionally, these cells accounted for more than half of the total tumor. Thus, our cases were diagnosed as pleomorphic adenomas, defined as tumors consisting of cells with a clear luminal and myoepithelial differentiation ${ }^{3}$.

\section{References}

1. Heath JE. Adenoma, adenocarcinoma, salivary gland, mouse. In: Monographs on Pathology of Laboratory Animals, Digestive System, 2nd ed., TC Jones, JA Popp and U Mohr (eds), Berlin: Springer, 236-239, 1997.

2. Betton GR, Whitely LO, Anver MR, Brown R, Deschl U, Elwell M, Germann PG, Hartig F, Kuttler K, Mori H, Nolte T, Puschner H, and Tuch K. Gastrointesitinal tract. In: International Classification of Rodent Tumors The Mouse, $\mathrm{U}$ Mohr (ed), Berlin: Springer, 23-58, 2001.

3. Cheuk W and Chan JKC. Salivary gland tumors. In: Diagnostic Histopathology of Tumors, CDM Fletcher (ed), London: Churchill Livingston, 236-243, 2000.

4. Ogawa Y, Yamauchi S, Ohnishi A, Ito R, and Ijuhin N. Immunohistochemistry of myoepithelial cells during development of the rat salivary glands. Anat Embryol (Berl) 200: 215-228, 1999.

5. Nagao T. Differential diagnosis of salivary gland tumors with the features of "Basaloid pattern" and "Cribriform Pattern”. Byori to Rinsho (in Japanese) 20: 23-26, 2002.

6. Head KW and Else RW. Other tumors of the alimentary tract. In: Tumors in Domestic Animals, 4th ed., DJ Meuten (ed), Ames, Iowa: Iowa State Press, 410-420, 2002. 